\title{
Europhysics Style Manual
}

\section{A guide to authors submitting papers to Europhysics Journals}

Foreword

This guide provides instructions to authors to help them prepare their papers in a way which minimises the number of editorial alterations required and hence the time needed for publication. It does not aim to provide advice on how to write clear, useful, concise papers. Such advice is provided by the IUPAP-Unesco Guide for the Preparation of Scientific Papers for Publication (IUPAP 14 or Unesco SC/MD/5) and to some extent by the Style Manual of the American Institute of Physics. (A recent guide, for the sciences generally, is O'Connor M. and Woodford F.P., Writing Scientific Papers in English (Elsevier, Amsterdam) 1975.

The recommendations made in this manual are in accordance with the general view of the editors of primary physics journals in Europe, especially those recognised by EPS as Europhysics Journals, but there are small variations in practice from publisher to publisher. Authors are advised to look at a recent number of the journal to which they are submitting a paper, and to refer to the journal's instructions to authors.

1. Material to be Submitted

The following material will be required before a paper can be prepared for the printer. Time will be saved if it is all submitted at the outset.

(i) Two copies of the typescript, each including:

Title page: with title of paper, author's (or authors') name(s) and establishment(s) where the work was done (present address(es), if different, should be given as a footnote).

Short title (if necessary) of not more than 50 letters for use at page head.

Abstract (on title page or separate sheet).

Text typed with double spacing on one side of A4 paper (210 mm x $297 \mathrm{~mm}$ ) with a margin of not less than $40 \mathrm{~mm}$ on the left-hand side of the page. List of references (on separate sheet).

List of captions for illustrations (on separate sheet).

Set of copies of illustrations (preferably A4 size) with lettering and figures included.

Set of tables (each table on a separate sheet).

(ii) One further copy of the title page and abstract.

(iii) One set of all line illustrations of a quality suitable for reproduction, with author's name in pencil. (Some publishers prefer no lettering or figures and other publishers lettering and figures in pencil only.)

(iv) One set of good-quality glossy prints of photographs, with author 's name in pencil in such a way as not to spoil the photograph.

(v) An English version of the title and abstract if the paper is not written in English.

(vi) Classification numbers, according to the International Classifi- 
The Guide has been prepared by the EPS Advisory Committee on Publications.

It is presented in a type-written form to avoid confusion with the subsequent printing operation. Further copies are available from the EPS Secretariat on request.

cation for physics (ICSU-AB, 1977), under which the paper should be indexed (see Appendix B). Numbers should always be given to two decimal points (for example 21.30), and should include the single letter indicating the fourth level of classification, where appropriate (e.g. 2l.6OF).

(vii) A covering letter, signed by one of the authors, and/or the officer responsible for publications in the establishment, requesting publication.

2. General Presentation

The paper must be typewritten on one side of the page only, in doublespacing and with a margin of $40 \mathrm{~mm}$. Good quality white bond paper of A4 size (210 mm x $297 \mathrm{~mm}$ ) should be used. Legible photocopies of the typescript are also acceptable. All pages should be numbered and the start of each paragraph should be clearly indicated.

Mathematics should be legible and authors should indicate by name in the margin any special type required (for example, Greek, Gothic, bold script) where it first occurs in the text. Authors should take particular care to distinguish scalar, vector and tensor quantities by underlining with a straight ( $\underline{V}$ scalar) or wavy $(\underset{\sim}{V}$ vector) or double wavy $(\underset{\approx}{\mathbb{V}}$ tensor) line respectively. Handwritten symbols and letters should be ̌ㅣearly written with marginal notes if any ambiguity exists (See Section 5.)

Section headings should be numbered with arabic numerals and placed at the left hand side of the page beginning with:

Introduction and continuing in the same fashion, including Conclusions; the Acknowledgements' section heading need not be numbered. Subsections, if used should appear as decimal derivatives, e.g., 2.1 and so on; subsections of these, if any, may be labelled 2.1.1, etc. Cross referencing should be by section or subsection number; page numbers should not be used.

Insertions in the paper should be typed on separate sheets and numbered (for example, $6 a$, with a note at the bottom of page 6 that it is to be followed by $6 a$, and a note at the bottom of page $6 a$ indicating on what page the text continues).

\section{Title Page}

The title and details of the authors should appear on a separate sheet, which may also include the abstract. The date of receipt of the typescript will be inserted by the editorial office.

If more than one paper with the same title and the same or different authors is submitted at the same time, the title should include a sub-title preceded by a Roman numeral.

The name(s) of the author(s) should be given below the title and followed by the name(s) of the establishment(s) where the work was done. The posi 
tal address(es), should be given. Present address(es), if different, should be indicated in a footnote. Acknowledgements of supporting organisations may be given in a footnote or in the "Acknowledgements' section.

4. Abstract

Each paper must have an abstract. It should be typewritten, like the body of the manuscript, and of a length not exceeding 38 of the text, or 250 words. The title should be regarded as part of the abstract but the abstract should otherwise be self-contained, not quoting the figures or references. It should be clear, concise and informative, giving the scope, the main results obtained and, for experimental papers, some indication of the methods used, where this is not obvious. Examples of good and bad abstracts are:

Good: Absorption measurements for the cadmium vapour give cross-sections of 7.9 and $12 \times 10^{-18} \mathrm{~cm}^{2}$ ( $1 \mathrm{no}$; at 50,70 and $100 \mathrm{~nm}$. These $\mathrm{nm}$ values are not in agreement with those of 20,25 and $35 \times 10^{-18} \mathrm{~cm}^{2} \mathrm{cal}-$ culated by R.G. Smith from a dipole formula.

Bad: The absorption of cadmium has been measured in the range 100 to $50 \mathrm{~nm}$, and the results are not in agreement with theory.

If the language of the paper is other than English, an English version of the abstract should also be given, the first line of which should be an English version of the title.

General guidance on the preparation of abstracts is given in the Unesco document $\mathrm{SC} / \mathrm{MD} / 5$.

\section{Mathematics}

Displayed equations must be clearly written each on a different line, well separated from the adjacent text. They should be numbered on the right hand side either consecutively through the text or with a compound number (for example, 3.15) which contains the section number. Particular care should be taken about the following points:

(i) A clear distinction, particularly in handwritten mathematics, must be made between similar letters and symbols.

Also note < (less than) and < (bracket) and the use of underlining to indicate italic script.

(ii) The use of brackets to indicate clearly what part of an expression should form the denominator of a fraction or be raised to a particular power. Brackets should be used in the order $\left\{\left[\begin{array}{ll}\text { ( ) } \\ \text { w }\end{array}\right.\right.$

(iii) The clear indication of indices, superscripts and subscripts.

(iv) The avoidance wherever possible of subscripts and superscripts to indices, subscripts and superscripts.

The form $\exp (\ldots)$ is preferred for exponentials unless they are simple ones; for example, $e^{x}$ but $\exp \left[(x+y)^{-1}\right]$.

For simple fractions in the text the solidus should be used (for example, $\pi / 7$ rather than $\frac{\pi}{7}$ ) but the proper fractions $\frac{1}{2}, 1 / 3,2 / 3$ are available as single types so that $(\lambda / 2) \pi$ may be given as $2 \lambda \lambda$. 
In displayed formulae

$$
\frac{6+5 \underline{h}+\underline{g}}{3 \underline{y z^{2}}}
$$

is better than

$$
(6+\underline{\underline{h}}+\underline{g}) / 3 \underline{y z}{ }^{2}
$$

and

$$
\frac{\underline{a} / \underline{b}+\underline{c} / \underline{d}+\underline{a} / \underline{d}}{\left(\underline{a}^{2}+\underline{b}^{2}\right)\left(\underline{c}^{2}+\underline{d}^{2}\right)}
$$

is better than

$$
\left(\frac{\underline{\mathrm{a}}}{\underline{\mathrm{b}}}+\frac{\underline{\mathrm{c}}}{\underline{\mathrm{d}}}+\frac{\underline{\mathrm{a}}}{\underline{\mathrm{d}}}\right) /\left(\underline{\mathrm{a}}^{2}+\underline{\mathrm{b}}^{2}\right)\left(\underline{\mathrm{c}}^{2}+\underline{\mathrm{d}}^{2}\right)
$$

If complicated expressions are repeated a number of times, single symbols should be used to define them on the first appearance and those used thereafter. For $\log _{e}$, use $\mathrm{ln}$; for $\log _{10}$ use $\mathrm{lg}$. A zero should always precede a decimal fraction (for example 0.123, or 0,123 in non-English text). For roots of anything other than a simple symbol or number, fractional superscripts are better than radical signs.

Average values of quantities other than those given by single symbols are best given as

$$
\left\langle\underline{x}^{2}+\underline{y}^{2}\right\rangle \text { av not } \underline{x}^{2}+\underline{y}^{2}
$$

Composite numbers should be expressed as $\mathrm{n} x \mathrm{~m}$ and not $\mathrm{n} . \mathrm{m}$.

\section{Illustrations}

Figures, whether diagrams, graphs or photographs, should be numbered consecutively in arabic numerals in the order in which they are mentioned in the text and this number together with the author's name should be written in pencil in the top right-hand corner of diagrams and on the back of photographs.

The fair copies of diagrams for reproduction should be in Indian ink on tracing paper, linen, white card or plastic and of sufficient size to permit reduction in printing. Very large figures should be avoided; unless there is a special reason, they should not be larger than A4.

\begin{tabular}{|c|c|c|c|}
\hline \multirow{2}{*}{ Reduction } & \multirow{2}{*}{$\begin{array}{l}\text { Minimum line } \\
\text { thickness in } \\
\text { axes and } \\
\text { curves } t / \mathrm{mm}\end{array}$} & \multicolumn{2}{|c|}{ Letters and symbols } \\
\hline & & $\begin{array}{l}\text { Height } \\
\text { H/mm }\end{array}$ & $\begin{array}{l}\text { Thickness } \\
\underline{t} / \mathrm{mm}\end{array}$ \\
\hline$\frac{1}{2}$ & 0.2 & 4 & 0.4 \\
\hline $1 / 3$ & 0.3 & 5 & 0.5 \\
\hline $1 / 4$ & 0.4 & 7 & 0.7 \\
\hline
\end{tabular}
Reductions to $\frac{1}{2}, 1 / 3$ and $1 / 4$ of original size are commonly used and the size of figures should allow for a suitable accommodation to the journal page size using one of these ratios.

Sizes of lines, axes, symbols and letters must comply with the following table: 
Inscriptions on drawings should be kept to a minimum and all explanations of different types of curve or data points should be incorporated in the caption. It is best to label curves with single symbols or with identifying letters. The detailed grid of a graph should not be reproduced, but grid lines at major multiples of units may be shown.

Notations of quantities and their numerical values on the axes must be made with care, in either of the following ways:

(i) The physical quantity is divided by its units to give a numerical value. Simple examples are $\mathrm{e} / \Omega \mathrm{cm}$ and $\mathrm{T} / \mathrm{K}$. Derived quantities are treated in the same way; for example, $\underline{C}_{\mathrm{V}} \mathrm{T}^{-1} / \mathrm{mJ} \mathrm{mol}^{-1} \mathrm{~K}^{-2}$ (see Figure 1 ). Symbols or combinations of symbols are shown to the left of the solidus, and units to the right.

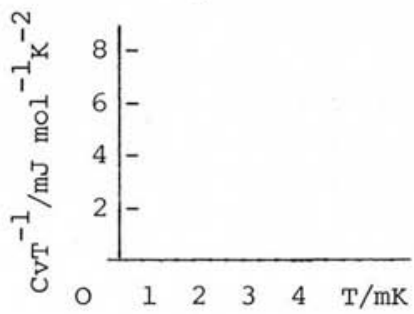

Figure 1

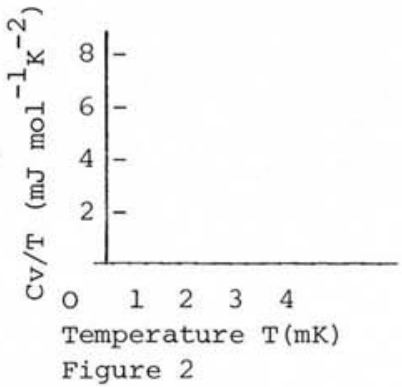

or (ii) The units are given in brackets after the physical quantity (see Figure 2).

Large or small numbers can be indicated by placing the appropriate power of ten, with a multiplication sign, near one of the numbers of the axis (for example, $\times 10^{6}$ or $\times 10^{-3}$ ). However, it is preferable to use the appropriate decimal multiplier (for example, $\mu \Omega, \mathrm{MJ}, \mathrm{nV}, \mathrm{GHz}$ ). In case of doubt or difficulty, a full explanation should be given in the caption.

Essential photographs can be reproduced but they should be avoided wherever possible; they should be on glossy paper, unmounted, with as much contrast as possible. Magnifications for photomicrographs should be indicated by a scale mark on the print, and the orientation of photographs should be indicated (for example, by TOP7 if any ambiguity exists. Line diagrams of apparatus should be schematic and detailed dimensions should not appear in the figure.

Captions must not be written at the bottom of figures but typed in order on a separate page.

If figures are reproduced from other articles the source must be quoted.

7. Tables

Each table must be typed on a separate sheet and labelled at the top with consecutive arabic or Roman numerals and as concise a title as possible. Even small amounts (4 lines) of tabular material should be treated in this way.

Headings for columns in tables should be brief and should use the same notation as that chosen in $\$ 6$ for the notations on the axes of figures. 
Footnotes to tables should be indicated by lower case superscript letters and listed below the table.

References for data in tables may be given in full in footnotes or indicated in the footnote in the manner used for references in the text.

Guidance on the presentation of numerical data in the primary literature can be obtained from CoDATA, 51 Boulevard de Montmorency, F-75016, Paris.

8. Footnotes and References

Footnotes should be indicated by a dagger ${ }^{t}$, asterisk ${ }^{*}$, etc., in the text and typed between two horizontal lines immediately after the line of text containing this indication.

References to previous work are made in the text with superscript arabic numerals and a following bracket, in order of their appearance (e.g., Smith and Jones 7 ) have shown.... or, it has been shown ${ }^{7}$ ) .....)*

Footnote:

*The Philosophical Magazine, Astronomy and Astrophysics and the Journals of Physics use the Harvard system, in which reference in the text is by author and year (e.g., Smith and Jones (1972) have shown....., and the list of references is in alphabetical order of authors' names.

The names of authors of references should be given in the text; et al. should not be used for less than three authors. The names and initials of all authors should be given in the list of references.

The list of references must be typed on a separate sheet in order of their appearance in the texts. Journal references should be in the form:

5. Smith S. and Jones J.H., Phys.Rev. 295 (1972) 422-30,

and book references in the form:

7. Carroll L., Introductory Nuclear Physics (Cavendish Press, Oxford) 1953 pp 50-63.

Abbreviations of periodical titles should conform to those recommended by member societies of ICSU-AB. The form given in the list of journals abstracted by Physics Abstracts is close to that used by most journals. If the author is in any doubt it is best to leave the title in full. The publishers, town and year of publication should be given for books, conference proceedings, etc., together with the name(s) of any editor(s). Unpublished conferences should not be given in the reference list. Papers in course of publication should be entered only if the journal has accepted them and its name is given. A thesis submitted and approved for a higher degree at a University may be listed. Unpublished work should not be given in the references but mentioned only in the text, e.g. (H. Marcuse 1969, personal communication) or (O. Welles, unpublished). The name of the institute of authors whose personal communications are quoted would be helpful. Internal laboratory reports not available on request should not be quoted; those available may be. Whenever possible, references to Russian literature should give the original and any translated version. 
Appendix A: Symbols, Units and Nomenclature

Authors are encouraged to follow the recommendations of the Document UIP 11 (SUN 65-3) 1965, of the International Union of Pure and Applied Physics and any later IUPAP recommendations. Document UIP 11 (SUN 65-3) 1965 may be obtained from Professor J.S. Nilsson, Associate Secretary General, Institute of Theoretical Physics, Fack S-40220, Göteborg 5, Sweden.

Authors' attention is drawn to the International System of Units. Up-todate details are given in Système International d'Unités, 1977, OFFILIB, 48 Rue Gay Lussac, F-75005 Paris, France (English translation SI-The International System of Units, 1977, London, HMSO).

Appendix B: ICSU/AB International Classification for Physics

Summary of scheme, see Physics Abstracts for details.

OO. OO GENERAL

01.00 Communication, education, history and philosophy.

02.00 Mathematical methods in physics.

03.00 Classical and quantum physics: mechanics and fields.

04.00 Relativity and gravitation.

05.00 Statistical physics and thermodynamics.

06.00 Measurement science, general laboratory techniques and instrumentation systems.

07.00 Specific instrumentation and techniques of general use in physics.

10.00 PHYSICS OF ELEMENTARY PARTICLES AND FIELDS

11.00 General theory of fields and particles.

12.00 Specific theories and interaction models; particle systematics.

13.00 Specific reactions and phenomenology.

14.00 Properties of specific particles and resonances.

20.00 NUCLEAR PHYSICS

21.00 Nuclear structure.

23.00 Nuclear decay and radioactivity.

24.00 Nuclear reactions and scattering: general.

25.00 Nuclear reactions and scattering: specific reactions.

27.00 Properties of specific nuclei listed by mass ranges.

28.00 Nuclear engineering and nuclear power studies.

29.00 Experimental methods and instrumentation for elementary-particle and nuclear physics.

30.00 ATOMIC AND MOLECULAR PHYSICS

31.00 Electronic structure of atoms and molecules: theory.

32.00 Atomic spectra and interactions with photons.

33.00 Molecular spectra and interactions with photons.

34.00 Atomic and molecular collision processes and interactions.

35.00 Experimentally derived information on atoms and molecules; instrumentation and techniques.

36.00 Studies of special atoms and molecules.

40.0O CLASSICAL AREAS OF PHENOMENOLOGY

41.00 Electricity and magnetism; fields and charged particles.

42.00 Optics. 
43.00

44.00

46.00

47.00

50.00

51.00

52.00

60.00

61.00

62.00

63.00

64.00

65.00

66.00

67.00

68.00

70.00

71.00

72.00

73.00

74.00

75.00

76.00

77.00

78.00

79.00

80.00

81.00

82.00

87.00

90.00

91.00

92.00

93.00

94.00

95.00

96.00

97.00

98.00

Acoustics.

Heat flow, thermal and thermodynamic processes.

Mechanics, elasticity, rheology.

Fluid dynamics.

FLUIDS, PLASMAS, AND ELECTRIC DISCHARGES

Kinetic and transport theory of fluids; physical properties of gases.

The physics of plasmas and electric discharges.

CONDENSED MATTER; STRUCTURE, THERMAL AND MECHANICAL PROPERTIES. Structure of liquids and solids; crystallography.

Mechanical and acoustic properties of condensed matter. Lattice dynamics and crystal statistics.

Equations of state, phase equilibria, and phase transitions. Thermal properties of condensed matter.

Transport properties of condensed matter (non-electronic). Quantum fluids and solids; liquid and solid helium.

Surfaces and interfaces; thin films and whiskers.

CONDENSED MATTER: ELECTRONIC STRUCTURE, ELECTRICAL, MAGNETIC, AND OPTICAL PROPERTIES.

Electron states.

Electronic transport in condensed matter.

Electronic structure and electrical properties of surfaces, interfaces, and thin films.

Superconductivity.

Magnetic properties and materials.

Magnetic resonances and relaxation in condensed matter; Mössbauer effect.

Dielectric properties and materials.

optical properties and condensed matter spectroscopy and other interactions of matter with particles and radiation.

Electron and ion emissions by liquids and solids; impact phenomena.

CROSS-DISCIPLINARY PHYSICS AND RELATED AREAS OF SCIENCE AND TECHNOLOGY

Materials science.

Physical chemistry.

Biophysics, medical physics and biomedical engineering.

GEOPHYSICS, ASTRONOMY AND ASTROPHYSICS.

Solid earth geophysics.

Hydrospheric and atmospheric geophysics.

Geophysical observations, instrumentation and techniques.

Aeronomy and space physics.

Fundamental astronomy and astrophysics, instrumentation and techniques and astronomical observations.

Solar system.

Stars

Stellar systems; galactic and extragalactic objects and systems; the universe. 